\title{
Collective vortex phases in periodic plus random pinning potential
}

\author{
W. V. Pogosov, ${ }^{1,2}$ V. R. Misko, ${ }^{1}$ H. J. Zhao, ${ }^{1}$ and F. M. Peeters ${ }^{1}$ \\ ${ }^{1}$ Departement Fysica, Universiteit Antwerpen, Groenenborgerlaan 171, B-2020 Antwerpen, Belgium \\ ${ }^{2}$ Institute for Theoretical and Applied Electrodynamics, Russian Academy of Sciences, Izhorskaya 13, 125412 Moscow, Russia
}

(Received 30 June 2008; published 6 January 2009)

\begin{abstract}
We study theoretically the simultaneous effect of regular and random pinning potentials on the vortex lattice structure at filling factor of 1 . This structure is determined by a competition between the square symmetry of regular pinning array, by the intervortex interaction favoring a triangular symmetry, and by the randomness trying to depin vortices from their regular positions. Both analytical and molecular-dynamics approaches are used. We construct a phase diagram of the system in the plane of regular and random pinning strengths and determine typical vortex lattice defects appearing in the system due to the disorder. We find that the total disordering of the vortex lattice can occur either in one step or in two steps. For instance, in the limit of weak pinning, a square lattice of pinned vortices is destroyed in two steps. First, elastic chains of depinned vortices appear in the film; but the vortex lattice as a whole remains still pinned by the underlying square array of regular pinning sites. These chains are composed into fractal-like structures. In a second step, domains of totally depinned vortices are generated and the vortex lattice depins from regular array.
\end{abstract}

DOI: 10.1103/PhysRevB.79.014504

PACS number(s): 74.25.Qt

\section{INTRODUCTION}

Magnetic and current-carrying properties of superconducting films with nanoengineered arrays of periodic and quasiperiodic pinning sites attract a lot of attention from both the experimental ${ }^{1-11}$ and theoretical ${ }^{12-18}$ points of view. The main reason of this interest is that the regularity in positions of pinning sites produces a large increase in the critical current at certain values of the magnetic field which correspond to the matching between the number of vortices and the number of pinning sites in the system. The highest value of the critical current in films with periodic pins was obtained for the same concentration of vortices and pinning sites. If the pinning potential is strong enough, vortices in a film with a square array of pinning sites also form a square lattice instead of the triangular one, the latter being energetically favorable in the absence of pinning. This regime corresponds to single-vortex pinning when vortices are pinned individually and the flux-line lattice behaves not as a collective media. However, if the pinning strength becomes on the order of the elastic energy of the flux-line lattice, the vortex-vortex interaction becomes important and the triangular symmetry of the vortex array is recovered. Thus, at the filling factor of 1 , low values of the pinning strengths favor a deformed triangular vortex lattice, which is basically depinned from the pinning array. At high values of the pinning strength, the regular square array of pinned vortices is the lowest-energy state. There is also an intermediate regime, where the vortex lattice is regular, but half of the vortex rows are depinned and form a lattice with a symmetry close to the triangular one. These three vortex phases are depicted in Fig. 1.

Structural phase transitions due to the tuning of the regular pinning strengths were studied theoretically within the framework of the London theory ${ }^{19-21}$ where vortices were treated as pointlike objects, i.e., near the first critical field. The same regime was considered in Refs. 22 and 23 by using molecular-dynamics simulations. Reference 24 analyzes such transitions near the second critical field using the linearized
Ginzburg-Landau theory, whereas in Refs. 16, 17, and 25 the whole system of the Ginzburg-Landau equations was solved numerically. There are also applications of the same ideas for the vortex lattices in rotating Bose-Einstein condensates of alkali-metal atoms, where regular pinning potential can be formed and easily tuned, in a broad range, by using lasers. At first, structural transitions of vortex lattices in this system were predicted theoretically in Refs. 26-28 using GrossPitaevskii equation and then observed experimentally. ${ }^{29} \mathrm{We}$ also would like to mention a recent experimental work ${ }^{30}$ on elastic lattices of millimeter-size charged particles in a square array of traps which exhibit similar physics as the here-considered vortex phases. Another example is colloidal particles on periodic substrates. ${ }^{31,32}$ Thus, the problem of the competition between the symmetry of the underlying pinning array and the lattice of repealing particles is important in various fields of modern physics. Note that in the case of superconducting films, holes which are usually used to create regular pins produce a strong pinning potential, which always dominate the vortex-vortex interaction. Weak enough pinning sites can be fabricated, for example, by imposing a periodic modulation on the film surface and were recently studied theoretically in Ref. 25.

\begin{tabular}{|c|c|c|c|c|c|c|c|c|c|c|c|}
\hline 0 & 0 & $\circ$ & $0^{(a)}$ & $\circ$ & 0 & $\odot$ & $0^{(b)}$ & - & - & - & $0^{(c)}$ \\
\hline & 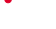 & 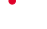 & $\bullet$ & & • & & • & & & & \\
\hline 0 & $\infty$ & 0. & $\because$ & $\odot$ & $\begin{array}{l}0 \\
\text {. }\end{array}$ & $\odot$ & $\begin{array}{l}0 \\
\text {. }\end{array}$ & • & - & - & ๑ \\
\hline 0 & . 0 & - 0 & .0 & $\odot$ & $\begin{array}{l}0 \\
\text {. }\end{array}$ & $\odot$ & $\begin{array}{l}0 \\
\text {. }\end{array}$ & $\circ$ & 0 & • & - \\
\hline - & $\bullet$ & - & $\infty$ & ○ & 0 & $\odot$ & O & - & - & - & $-0_{x}$ \\
\hline
\end{tabular}

FIG. 1. (Color online) Vortex lattice structure in a superconducting film with weak periodic pinning at filling factor of 1 . Vortices are shown by red (dark gray) filled circles and pinning sites by black open cirles. (a) corresponds to the case of very low pinning with the deformed triangular vortex lattice. (b) shows a half-pinned regular phase realized at intermediate values of pinning strength. (c) represents a pinned vortex lattice, which is energetically favorable at higher values of pinning strength. 
In the present paper, we consider theoretically a more complicated situation, when there is an additional factor, namely, a random pinning potential. Random pinning is always found in any real physical system; moreover randomness cannot be ruled out even in numerical simulations, where its effect should be carefully analyzed. Samples potentially important for applications have to be very large compared to the period of the regular pinning potential and, therefore, disorder and defects in lattices of vortices or other interacting objects are unavoidable. For this reason, understanding of basic properties of such systems is of scientific and hopefully of practical importance.

Thus the structure of the vortex lattice in the system under consideration is determined by three factors: (i) a square array of regular pinning sites, which tries to impose its own symmetry on the vortex lattice; (ii) an interaction between vortices, which favors a triangular configuration of the vortex lattice; and (iii) a random pinning potential, which attempts to destroy the regularity in the vortex positions and to depin them from the square lattice. We analyze here analytically various kinds of vortex lattice defects for a twodimensional system and determine typical values of the random pinning strengths leading to a spontaneous generation of these defects. This allows us to construct an approximate phase diagram of the system in the plane of regular pinning strength and random pinning strength. We also perform molecular-dynamics simulations in order to model numerically the process of disordering of the square vortex array. The obtained vortex patterns and the typical values of random pinning strength producing disordering are in good agreement with our expectations obtained from the analytical treatment.

Note that the problem studied can be also linked to other situations when there is a competition of randomness and regularity, but where the dimensionalities of the pinning potential and an elastic media are possibly different from two dimensional. These are flux-line lattices in layered superconductors, where layers act as one-dimensional (1D) pinning centers giving rise to a so-called intrinsic pinning. Such pinning centers can also be created by twin boundaries. Since there is always disorder in real superconductors, one can also have a competition between randomness and regularity. ${ }^{33-35}$ Other examples are charge- and spin-density waves.

The paper is organized as follows. In Sec. II, we present the basic formulation of our model and discuss our approach of molecular-dynamics simulations. In Sec. III, various kinds of kinks or defects in vortex lattices are found, their structures are discussed, and their energies and sizes are estimated. In Sec. IV, we characterize a random potential and explain how to account for its effect on vortex lattices. Our main results are given in Sec. V, where we construct an approximate phase diagram of the system in the plane of regular and random pinning strengths. Finally, we conclude in Sec. VI.

\section{MODEL}

\section{A. Basic formalism}

In our model, we treat vortices as pointlike objects interacting via a pairwise potential, which are valid assumptions as long as the applied magnetic field is much lower than the second critical field $H_{c 2}$ and the Ginzburg-Landau parameter is large, $\kappa \gg 1$. Under such assumptions, the interaction energy of two vortices positioned at $\mathbf{r}_{1}$ and $\mathbf{r}_{2}$ can be well approximated by the well-known London expression,

$$
H_{\text {int }}\left(\mathbf{r}_{1}, \mathbf{r}_{2}\right)=\frac{2 \pi}{\kappa^{2}} K_{0}\left(\left|\mathbf{r}_{1}-\mathbf{r}_{2}\right|\right),
$$

where $K_{0}$ is a modified Bessel function. Here and below the following dimensionless variables are used: distances are measured in terms of the London penetration depth $\lambda$ and energy is measured in units of $H_{c}^{2} \lambda^{3} / 8 \pi$, with $H_{c}$ being the thermodynamic critical field. An important quantity, which we are going to use, is the interaction energy of a regular vortex row with a given vortex situated outside of this row. If we put a center of coordinates at one of the vortices in the row, and the $y$ axis is along the row, then the interaction energy of the vortex located at $(x, y)$ is given by

$$
H_{\mathrm{int}}^{\mathrm{row}}(x, y)=\frac{2 \pi}{\kappa^{2}} \sum_{m=-\infty}^{\infty} K_{0}\left(\sqrt{x^{2}+(y+m d)^{2}}\right),
$$

where $d$ is the intervortex distance. Using Fourier transformation for a modified Bessel function $K_{0}(r)$ and performing a summation in the reciprocal space, Eq. (2) can be rewritten as

$$
\begin{aligned}
H_{\text {int }}^{(\text {row })}(x, y)= & \frac{2 \pi}{\kappa^{2}} \frac{\pi}{d} \sum_{m=-\infty}^{\infty} \frac{1}{\sqrt{1+\frac{4 \pi^{2} m^{2}}{d^{2}}}} \\
& \times \exp \left(-x \sqrt{1+\frac{4 \pi^{2} m^{2}}{d^{2}}}\right) \cos \frac{2 \pi m y}{d} .
\end{aligned}
$$

Vortices are located in a two-dimensional film with a periodic square array of pinning sites. To describe the pinning potential of a single site, we use a parabolic function,

$$
V(\mathbf{r})=-U_{0}\left[1-\left(\frac{|\mathbf{r}|}{\sigma}\right)^{2}\right]
$$

inside the well, $r \leq \sigma$, and the potential is zero outside the well, $r>\sigma$. In this paper, we consider the situation when the well radius $\sigma$ is much smaller than the intersite distance $a$, i.e., $\sigma \ll a$.

In addition to the regular pinning potential there is also a random pinning potential in the system; we denote its energy by $\varepsilon(\mathbf{r})$. The total energy of the system is thus given by

$$
E=\sum_{i, j} H_{\mathrm{int}}\left(\mathbf{r}_{i}, \mathbf{r}_{j}\right)+\sum_{i, n} V\left(\mathbf{R}_{n}-\mathbf{r}_{i}\right)+\sum_{i} \varepsilon\left(\mathbf{r}_{i}\right),
$$

where $\mathbf{r}_{i}$ and $\mathbf{R}_{n}$ stand for the positions of vortices and regular pinning sites, respectively. We also assume that the number of vortices is exactly equal to the number of pinning sites, i.e., filling factor is 1 .

Let us recall first the situation when there is no random potential in the system. In this case, as was explained in Sec. I, strong regular pinning favors a square vortex lattice symmetry [see Fig. 1(c)]. At low $U_{0}$ the repulsion between vortices dominates resulting in a slightly deformed triangular 
lattice that has the lowest energy, as illustrated in Fig. 1(a). Finally, in the intermediate range of $U_{0}$, a half-pinned lattice becomes the ground state [see Fig. 1(b)]. In the half-pinned phase, an effective pinning potential for depinned vortices is created by their interaction with neighboring rows of pinned vortices. It is easy to see that the following expressions define the boundaries between the triangular lattice and the half-pinned phase and half-pinned phase and square lattice, respectively: ${ }^{19,21}$

$$
\begin{aligned}
& U_{0} \simeq 2\left(E_{\mathrm{hp}}-E_{\mathrm{tr}}\right), \\
& U_{0}=2\left(E_{\mathrm{sq}}-E_{\mathrm{hp}}\right),
\end{aligned}
$$

where $E_{\mathrm{tr}}, E_{\mathrm{hp}}$, and $E_{\mathrm{sq}}$ are the energies of triangular, halfpinned, and square vortex lattices, without any pinning, taken per one vortex. In other words, these vortex-vortex interaction energies depend only on vortex lattice symmetry. We focus on the situation when the strength of a regular pinning is not too high, i.e., is close to the boundaries defined by Eqs. (6) and (7). In this case the vortex lattice can be considered as a collective object and we are far from the regime of single-vortex pinning.

\section{B. Molecular-dynamics simulations}

In addition to an analytical approach, we supplement our study with computer experiments. In this section, we describe briefly the basic ingredients of the used computer simulations. We use molecular-dynamics simulations of vortices moving under the action of the forces due to the vortexvortex interaction and the interaction of vortices with regular and random pinning sites. To find the lowest-energy vortex configurations, we perform simulated annealing simulations by numerically integrating the overdamped equations of motion (see, e.g., Refs. 12-15 and 18),

$$
\eta \mathbf{v}_{i}=\mathbf{f}_{i}=\mathbf{f}_{i}^{v v}+\mathbf{f}_{i}^{v p}+\mathbf{f}_{i}^{T}
$$

Here, $\mathbf{f}_{i}$ is the total force per unit length acting on vortex $i$, $\mathbf{f}_{i}^{v v}$ and $\mathbf{f}_{i}^{v p}$ are the forces due to the vortex-vortex and vortexpin interactions, respectively, and $\mathbf{f}_{i}^{T}$ is the thermal stochastic force. In Eq. (8), $\eta$ is the viscosity, which is set here to unity. The force due to the interaction of the $i$ th vortex with other vortices is

$$
\mathbf{f}_{i}^{v v}=\frac{2 \pi}{\kappa^{2}} \sum_{j \neq i}^{N_{v}} K_{1}\left(\left|\mathbf{r}_{i}-\mathbf{r}_{j}\right|\right) \hat{\mathbf{r}}_{i j}
$$

where $N_{v}$ is the number of vortices, $K_{1}$ is the modified Bessel function, and $\hat{\mathbf{r}}_{i j}=\left(\mathbf{r}_{i}-\mathbf{r}_{j}\right) /\left|\mathbf{r}_{i}-\mathbf{r}_{j}\right|$. The modified Bessel function $K_{1}(r)$ decays exponentially for $r$ larger than 1 ( $\lambda$ in dimensional units), thus it is safe to cut off the (negligible) force for distances larger than 5. In our calculations, the logarithmic divergence of the vortex-vortex interaction forces for $r \rightarrow 0$ is eliminated by using a cutoff at distances smaller than 0.1 .

The force due to the interaction of the $i$ th vortex with the parabolic pinning potentials [Eq. (4)] is

$$
\mathbf{f}_{i}^{v p}=\sum_{k}^{N_{p}}\left(\frac{f_{p}}{\sigma}\right)\left|\mathbf{r}_{i}-\mathbf{r}_{k}^{(p)}\right| \Theta\left(r_{p}-\left|\mathbf{r}_{i}-\mathbf{r}_{k}^{(p)}\right|\right) \hat{\mathbf{r}}_{i k}^{(p)},
$$

where $N_{p}$ is the number of pinning sites, $f_{p}$ is the maximal pinning force of each potential well, $\sigma$ is the spatial range of the pinning potential (for random potentials, we define $f_{p r}$ $\lesssim f_{p}$ and $\sigma_{r} \lesssim \sigma$ as the maximum pinning force and radius, correspondingly), $\Theta$ is the Heaviside step function, and $\hat{\mathbf{r}}_{i k}^{(p)}$ $=\left(\mathbf{r}_{i}-\mathbf{r}_{k}^{(p)}\right) /\left|\mathbf{r}_{i}-\mathbf{r}_{k}^{(p)}\right|$.

The temperature contribution to Eq. (8) is modeled by a stochastic term obeying the following conditions:

$$
\left\langle f_{i}^{T}(t)\right\rangle=0
$$

and

$$
\left\langle f_{i}^{T}(t) f_{j}^{T}\left(t^{\prime}\right)\right\rangle=2 \eta k_{B} T \delta_{i j} \delta\left(t-t^{\prime}\right) .
$$

The ground state of a system of vortices is obtained as follows. First we set a high temperature to let vortices move randomly, then temperature is gradually decreased down to $T=0$, thus simulating field-cooled experiments (see, e.g., Refs. 36 and 37). Note that unlike in Refs. 12-15 and 18 we do not consider vortex dynamics driven by an external Lorentz force, but we only calculate the ground-energy vortex configuration (see, e.g., Refs. 38 and 39). In Sec. III we discuss the effect of a random potential on the square and the half-pinned phases and define typical vortex lattice defects induced by the randomness.

\section{KINKS}

Both square and half-pinned phases are periodic in the absence of random pinning sites. Translational motion of the vortex lattice as a whole by a period of a regular pinning lattice $a$ does not change the energy of the system. Therefore, one can expect that there exist such solutions when in one region of the system, the vortex lattice is shifted by $a$ with respect to that in another region. There should also be a kink with intermediate value(s) for deviations of vortex positions separating these domains. In this sense, our system resembles a traditional sine-Gordon system, which describes the behavior of elastic media in a periodic potential. ${ }^{40}$ For sine-Gordon systems, a typical kink has a smooth structure and its length depends both on the elastic properties of the media and the strength of the periodic potential, and it can be arbitrary large. Kinks are known to play an important role in the process of disordering of media. ${ }^{40}$ Thus one may say that disordering is occurring through a proliferation of kinks. Therefore, we will pay special attention to the kink structure.

\section{A. Continuous kinks versus sharp defects}

One can naively assume that there also exist smooth kinks for square-pinned and half-pinned lattices. However, despite the similarity with sine-Gordon systems, our system has one specific feature, namely, that square- and half-pinned vortex configurations are not the lowest-energy states in the absence of pinning. Moreover, they are locally unstable, i.e., even infinitesimally small displacements of vortices lead to the collapse of the lattice into a triangular array. In other words, 

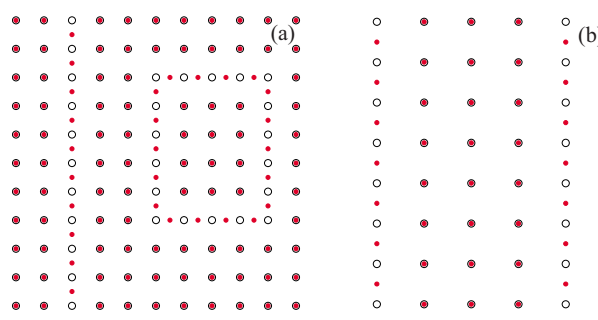

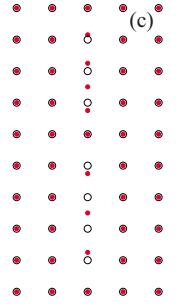

FIG. 2. (Color online) Schematic view of typical defects in a regular vortex lattice. Vortices or pinning sites are shown by red (dark gray) filled circles or black open cirles. (a) shows elastic strings of unpinned vortices in the square pinned lattice: straight (on the left) and reconnected (on the right). (b) presents a row of pinned vortices in the half-pinned phase. (c) corresponds to the quasi-onedimensional defect of finite length inside the pinned square lattice.

the elasticity theory does not work properly. This is shown explicitly in the Appendix, where we also determine a structure of an infinite-length kink. We found that it has a "discrete" (i.e., not smooth) structure, which is shown in Fig. 2(a). This is a fundamental difference between the system at hand and conventional sine-Gordon systems.

Note that in the infinite system this type of defects has a divergent energy because it is of infinitely long. Therefore, fluctuations generate reconnected kinks of finite sizes rather than infinitely long defects, as shown in Fig. 2(a). This point will be explained in detail in Sec. V. Also notice that the fact of appearance of reconnected defects is well known in the condensed-matter physics, see e.g., Ref. 40, for sine-Gordon and related systems.

It is important to notice that the string of unpinned vortices behaves as an elastic object; vortices inside the string are correlated and behave collectively due to their interaction. It is easy to see from Fig. 2(a) that such a defect can be considered as a nucleus of a half-pinned vortex phase. The defect energy density can be therefore estimated as a difference in the energy density between the half-pinned and pinned vortex phases. They are given, respectively, by

$$
\begin{gathered}
\varepsilon_{\mathrm{hp}}=\frac{E_{\mathrm{hp}}-U_{0} / 2}{a^{2}}, \\
\varepsilon_{\mathrm{sq}}=\frac{E_{\mathrm{sq}}-U_{0}}{a^{2}} .
\end{gathered}
$$

The first term in the numerator in the right-hand side (RHS) of Eq. (13) [Eq. (14)] is the energy of the vortex-vortex interaction, taken per one vortex, while the second term denotes the pinning energy per one vortex. The latter terms are different for the half-pinned [Eq. (13)] and for the pinned [Eq. (14)] phases since in the second case all the vortices are pinned, while in the first case-only half. Denominators in the RHS of Eqs. (13) and (14) give the area per one vortex. Finally, for the defect energy density we obtain

$$
E_{d}^{(1)} \approx \varepsilon_{\mathrm{hp}}-\varepsilon_{\mathrm{sq}}=\frac{U_{0} / 2-\left(E_{\mathrm{sq}}-E_{\mathrm{hp}}\right)}{a^{2}} .
$$

It is important to note that the kink energy vanishes at the transition between the square and half-pinned lattices, i.e., at
$U_{0}=2\left(E_{\mathrm{sq}}-E_{\mathrm{hp}}\right)$ since energies of these two phases become equal.

It is easy to realize that the pinned phase can also be disturbed by a domain composed by a region of a deformed triangular vortex lattice. Again such a domain is an elastic object; vortices within the domain are depinned collectively. The region of depinned vortices has higher-energy density compared to the previously described stringlike defect, since the energy of a deformed triangular lattice has a higher energy than the half-pinned lattice in the relevant parameter region. This energy density can be estimated in a similar manner, as the one which led us to Eq. (15). For that we need to know the energy density of the deformed triangular lattice, which is given by ${ }^{21}$

$$
\varepsilon_{\mathrm{tr}} \approx \frac{E_{\mathrm{tr}}}{a^{2}},
$$

where $E_{\mathrm{tr}}$ is the vortex-vortex interaction energy in the ideal triangular lattice taken per one vortex. In reality, this lattice is deformed and it is not ideal. However, as it was shown in Ref. 21, the energy of elastic distortions is compensated by the pinning energy in the linear approximation with respect to vortices displacement and Eq. (16) is correct. Finally, for the density energy of the defect, we obtain

$$
E_{d}^{(2)} \approx \varepsilon_{\mathrm{tr}}-\varepsilon_{\mathrm{sq}}=\frac{U_{0}-\left(E_{\mathrm{sq}}-E_{\mathrm{tr}}\right)}{a^{2}} .
$$

Now we turn to the description of the half-pinned phase. By similarity with the pinned phase, we can see that a typical kink in the half-pinned phase consists of a row of pinned phase. One can also have domains of triangular phases. The first type of defect is shown schematically in Fig. 2(b). The energy densities for these kinks can be calculated from the differences in energy densities of the nucleating phase and the initial one, as for Eqs. (15) and (17). They are given, respectively, by

$$
\begin{gathered}
E_{d}^{(3)} \approx \varepsilon_{\mathrm{sq}}-\varepsilon_{\mathrm{hp}}=\frac{-U_{0} / 2+\left(E_{\mathrm{sq}}-E_{\mathrm{hp}}\right)}{a^{2}}, \\
E_{d}^{(4)} \approx \varepsilon_{\mathrm{tr}}-\varepsilon_{\mathrm{hp}}=\frac{U_{0} / 2-\left(E_{\mathrm{hp}}-E_{\mathrm{tr}}\right)}{a^{2}},
\end{gathered}
$$

which of the kinks has a lower-energy density depends on the position in the phase diagram. As again can be expected, defects of the first type shown in Fig. 2(b) should be reconnected in finite systems.

\section{B. Quasi-one-dimensional defects}

We note that one more type of the defects appears in the system for sufficiently larger values of $U_{0}$, when the potential well for a pinned vortex row is much deeper than the effective potential well created for a depinned vortex row by surrounding rows of pinned vortices. In this limit, the defect considered is a finite row of unpinned vortices but is not reconnected. Such a structure can exist due to the fact that in one part of the defect, the string of vortices is stretched so that there is one missing vortex and the last vortex in this 
part of the string deviates from its equilibrium position by one period of the regular lattice $a$. In another part of the defect, the string of vortices is compressed so that there is an excess vortex. This defect is shown schematically in Fig. 2(c) for the most simple case when it is straight. It consists of a finite elastic string of vortices in the same row, which are displaced along the direction of the row. Thus this object as a whole can be considered as a quasi-one-dimensional (quasi-1D) kink-antikink structure. In Sec. V, by using molecular-dynamics simulations and rather general arguments, we argue that both reconnected chains of depinned vortices and quasi-one-dimensional defects are limiting cases of wider class of collective fractal-like defects. In other words, defects considered in this section are not necessarily straight, but straight defects can be more easily studied analytically.

We now calculate typical energy and length of this quasione-dimensional defect. We assume that positions of all other vortices in the system remain unchanged. The potential energy of a vortex in the string is given by the interaction energy with the fixed vortices, with pinning sites, and also with each other. The first contribution to the string energy can be found from Eq. (3), where one can neglect all the terms except of the ones with $m=0, \pm 1$ provided that $a$ $\sim 1$, which results in

$$
E_{1}^{(1 \mathrm{D})} \simeq \frac{4 \pi}{\kappa^{2}} \frac{\pi}{a} \sum_{k}\left(\frac{\exp (-a)}{1-\exp (-a)}+\frac{a}{\pi} \exp (-2 \pi) \cos \frac{2 \pi y_{k}}{a}\right),
$$

where the summation is performed over positions of all vortices in the string $y_{k}$. Note that from this equation one can see that the intervortex interaction favors a shift of $a / 2$ of the row in the $y$ direction with respect to the lattice making a symmetry closer to the triangular one. Note that potential energy (20) for each vortex has a typical sine-Gordon structure.

The interaction energy for the vortices with pinning sites in the row reads as

$$
E_{2}^{(1 \mathrm{D})}=\sum_{n=-\infty}^{\infty} \sum_{k} V\left(\text { an }-y_{k}\right)
$$

where $V(y)$ is defined by Eq. (4) and the index $n$ stands for the positions of the pinning sites. For illustrative purposes, we plot the sum of $E_{1}^{(1 \mathrm{D})}$ and $E_{2}^{(1 \mathrm{D})}$ in Fig. 3(a) for vortices in the string that are positioned periodically, $y_{k}=y+k a$. The resulting potential is, of course, a periodic function in $y$. The energy minimum for the string is attained for a square configuration due to our choice of parameters, i.e., for strong enough regular pinning. To show that this potential is of a quasi-one-dimensional form, we also plot it in threedimensional form in Fig. 3(b).

There is an additional contribution to the energy of the vortex, which is due to its interaction with other vortices in the string, i.e., the elasticity term. This contribution depends on the mutual positions of the vortices. We will take into account only the interaction with nearest neighbors, as is usually done in elasticity theory. Taking into account Eq. (2)

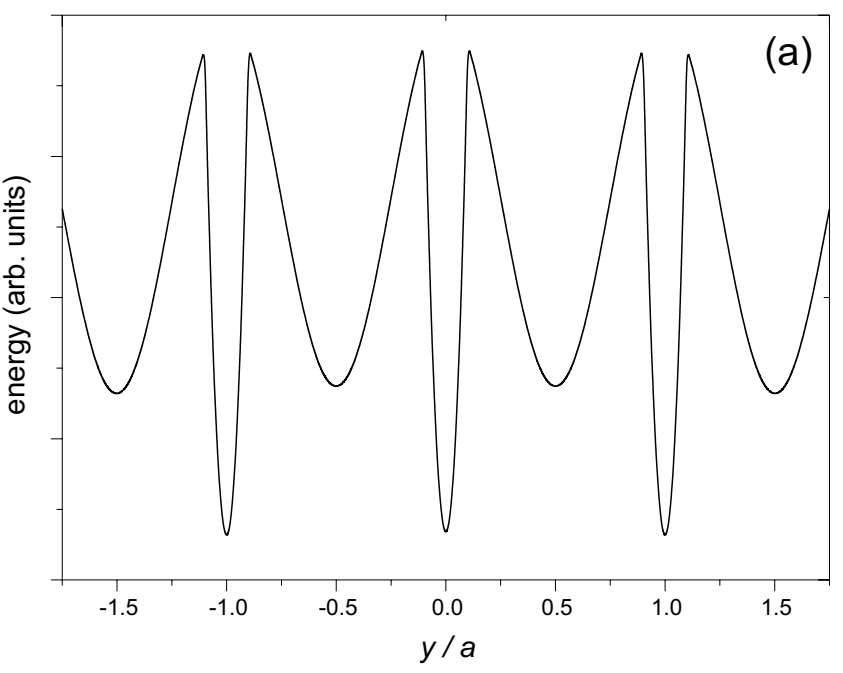

(b)

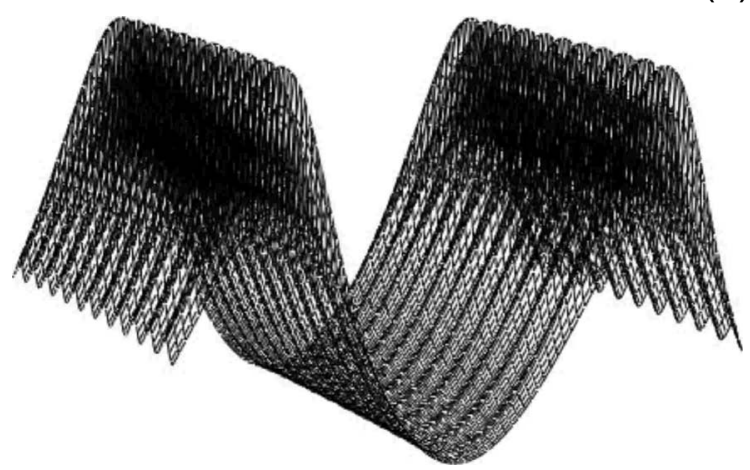

FIG. 3. Potential-energy profile for the vortex row in the square lattice along one of the principal axis provided that positions of vortices in the row are fixed with respect to each other and all other vortices are pinned. (a) corresponds to the dependence on one coordinate, whereas (b) shows a dependence on both coordinates, which is of quasi-one-dimensional form.

and using a Taylor expansion for the modified Bessel function, we obtain the following expression for the energy of the whole string of vortices:

$$
E_{3}^{(1 \mathrm{D})}=\frac{2 \pi}{\kappa^{2}}\left(K_{0}(a)+\frac{K_{1}(a)}{a}\right) \sum_{k}\left(\Delta u_{k}\right)^{2},
$$

where $\Delta u_{k}$ is the displacement of $k$ th vortex with respect to the $(k-1)$ th one. As usual, we can replace $\Delta u_{k} / a$ by $d u / d y$ and also switch from the summation to the integration in the RHS of Eq. (22), ${ }^{40}$

$$
E_{3}^{(1 \mathrm{D})}=\frac{2 \pi a}{\kappa^{2}}\left(K_{0}(a)+\frac{K_{1}(a)}{a}\right) \int\left(\frac{d u}{d y}\right)^{2} d y,
$$

where the integration is performed along the length of the defect. The total energy of the defect is thus given by the sum of three contributions:

$$
E^{(1 \mathrm{D})}=E_{1}^{(1 \mathrm{D})}+E_{2}^{(1 \mathrm{D})}+E_{3}^{(1 \mathrm{D})},
$$

which can be estimated in a simple manner. We assume that a typical kink-antikink structure has a length $l_{1 \mathrm{D}}$. There are $l_{1 \mathrm{D}} / a$ vortices along this defect and they are depinned from 
their lowest-energy positions. Each vortex gains some energy by being depinned. If the width of the potential well $\sigma$ is much smaller than the intersite distance $a$, the gain is given by $U_{0}$, as follows from Eq. (23). At the same time, each vortex also loses some amount of energy due to the fact that it no longer forms a square configuration with vortices from the surrounding fixed rows, as can be seen from Eq. (20). Thus, the total-energy increase for the string of $l_{1 \mathrm{D}} / a$ depinned vortices is given approximately by

$$
E_{1}^{(1 \mathrm{D})}+E_{2}^{(1 \mathrm{D})} \approx l_{1 \mathrm{D}} / a\left(U_{0}-\frac{4 \pi}{\kappa^{2}} e^{-2 \pi}\right) .
$$

The elastic energy of the string $E_{3}^{(1 \mathrm{D})}$ can be easily estimated from Eq. (23) as

$$
E_{3}^{(1 \mathrm{D})} \approx \frac{2 \pi a^{3}}{\kappa^{2}} \frac{1}{l_{1 \mathrm{D}}}\left(K_{0}(a)+\frac{K_{1}(a)}{a}\right) .
$$

If we now minimize the total defect's energy $E^{(1 \mathrm{D})}$ with respect to its length $l_{1 \mathrm{D}}$, we obtain

$$
l_{1 \mathrm{D}} \approx \sqrt{\frac{2 \pi a^{4}}{\left(U_{0}-\frac{4 \pi}{\kappa^{2}} e^{-2 \pi}\right) \kappa^{2}}\left(K_{0}(a)+\frac{K_{1}(a)}{a}\right)} .
$$

This leads to the following result for the defect energy:

$$
E^{(1 \mathrm{D})} \approx 2 \sqrt{\frac{2 \pi a^{2}\left(U_{0}-\frac{4 \pi}{\kappa^{2}} e^{-2 \pi}\right)}{\kappa^{2}}\left(K_{0}(a)+\frac{K_{1}(a)}{a}\right)},
$$

which has a nontrivial dependence on $U_{0}$. As we will see below, in the limit of weak pinning, quasi-1D defects have higher energies compared to previously analyzed ones. This can be explained by noticing that vortices in the string are situated neither exactly in a potential well induced by pinning sites nor in that created by the neighboring rows of vortices.

Note that a quasi-1D defect can have a slightly different structure, when the maximum deviation of vortex in the string from its equilibrium position is given by $a / 2$ but not by $a$. In this case, the position of the most strongly displaced vortex in the row is stabilized by the interaction with neighboring rows of vortices and not by a pinning site. Our estimates for length and energy of quasi-one-dimensional defect given by Eqs. (27) and (28), however, remain applicable also for such a defect.

\section{RANDOM POTENTIAL}

In this section, we characterize the random potential and describe how to deal with it in our problem. We assume that the random potential is created by randomly distributed pinning wells with size $\sigma_{r} \lesssim \sigma, a$ and depth $U_{r} \lesssim U_{0}$. The concentration of random sites is denoted by $n_{r}$ and we also assume that it is higher or equal to the concentration of regular pinning sites $n_{r} \gtrsim a^{-2}$.

It is well known that the actual pinning energy due to the random pinning is produced by fluctuations of the random potential in a given region of the system, i.e. by bunches of pins, and not by pinning potential averaged value. If we have a region of area $S_{0} \gg n_{r}^{-1}$, the distribution probability $P(N)$ for having $N$ random pinning sites within this region can be well described by a normal distribution,

$$
P(N)=\frac{1}{\sqrt{2 \pi}} \frac{1}{\sqrt{n_{r} S_{0}}} \exp \left[-\frac{\left(N-n_{r} S_{0}\right)^{2}}{2 n_{r} S_{0}}\right] .
$$

As can be expected, the averaged value of $N$ is given by

$$
\langle N\rangle=n_{r} S_{0},
$$

whereas the mean-square deviation reads as

$$
\sqrt{\left\langle N^{2}\right\rangle-\langle N\rangle^{2}} \simeq n_{r} S_{0} .
$$

The probability of having any number of random pinning sites larger than some definite value $N_{0}$ within the region $S_{0}$ can be found by integration $\int_{N_{0}}^{\infty} P(N) d N$, and this integral can be estimated as (provided that $N_{0}-n_{r} S_{0} \gtrsim \sqrt{n_{r} S_{0}}$ )

$$
\int_{N_{0}}^{\infty} P(N) d N \approx \frac{1}{\sqrt{2 \pi}} \frac{\sqrt{n_{r} S_{0}}}{N_{0}-n_{r} S_{0}} \exp \left[-\frac{\left(N_{0}-n_{r} S_{0}\right)^{2}}{2 n_{r} S_{0}}\right] .
$$

The probability for this event not to occur is, of course, 1 $-\int_{N_{0}}^{\infty} P(N) d N$. If we now have a larger region $S>S_{0}$, the probability that within $S$, one cannot find a region of area $S_{0}$ with number of random pinning sites larger than $N_{0}$ is estimated as $\sim\left[1-\int_{N_{0}}^{\infty} P(N) d N\right]^{S / S_{0}}$. Finally, the probability to find such a region is $1-\left[1-\int_{N_{0}}^{\infty} P(N) d N\right]^{S / S_{0}}$. From this analysis and from Eq. (32) it is easy to see that if $S \sim S_{0}$ and $\mid N_{0}$ $-n_{r} S_{0} \mid \sim \sqrt{n_{r} S_{0}}$, this probability is very close to 1 . This means that within the domain of area $S$ slightly larger than $S_{0}$, we can always find a bunch of pines, such that the pinning energy around this bunch (within region of area $S_{0}$ ) deviates by $\sqrt{n_{r} S_{0}}$ from its average value. This illustrates that the effective pinning energy within some region can be closely approximated by mean-square deviation of random pinning potential within this region, $U_{r} \sqrt{n_{r} S_{0}}$. This conclusion is well known in the physics of disordered flux-line lattices. ${ }^{41}$ The famous Larkin-Ovchinnikov theory is also based on the idea that the mean-square deviation of random pinning energy is balanced with the energy of lattice distortions within some domain.

Each kind of defects described in Sec. IV has its own typical energy and sizes. In order to estimate the random pinning strength leading to a proliferation of such defects, one can equate the defect energy with the mean-square deviation of the random pinning potential within the characteristic area of the defect. By doing this, one can study the phase diagram of the system in the plane of random and regular pinning sites strengths.

\section{PHASE DIAGRAM}

In the absence of random pinning, there are two phase boundaries separating the pinned square, half-pinned and deformed triangular lattices, as given by Eqs. (6) and (7). We 
now consider the effect of the random pinning potential. Note that a deformed triangular vortex lattice is aperiodic even in the absence of random pinning, since periods of vortex and regular pinning sites lattices are incommensurate. Therefore we consider disordering of square-pinned and half-pinned vortex lattices, which have a regular structure. As was already explained, the destruction of the order occurs via a proliferation of defects. Using the tools developed in Secs. III and IV, we can estimate the typical random pinning strengths leading to a generation of different defects identified in Sec. III. We do not expect that this will give us the full phase diagram, since we have found defects only in some simple and limiting cases. In order to obtain a deeper insight into the problem we will also use moleculardynamics simulations.

Let us start from the case of a square-pinned lattice. The lowest-energy defect in such a situation, among those we have found, is constructed from the elastic string of collectively depinned vortices, as explained in Sec. III. This row has to be reconnected because otherwise its energy is infinitely large ${ }^{40}$ (therefore, one should pay special attention to this point both in numerical and real experiments on finite arrays, since there it is easy to get just straight defects - their energies remain finite because the system sizes are finite). The radius of such a defect cannot be too small since the system is discrete. Therefore, the lowest-energy defect has a radius $R_{d}$ of the order of the intersite distance $R_{d} \sim a$ [see Fig. 2(a)]. Let us write $R_{d}$ as $a r_{d}$, where the dimensionless factor $r_{d}$ is on the order of $1, r_{d} \sim 1$. The energy of such a defect can be found from Eq. (15),

$$
E_{\mathrm{kink}} \sim 2 \pi r_{d}\left[U_{0} / 2-\left(E_{\mathrm{sq}}-E_{\mathrm{hp}}\right)\right]
$$

The area of this structure is just $\pi a^{2} r_{d}^{2}$. To find the random pinning strength leading to a proliferation of this particular kind of defects, we equate the energy of the defect $E_{\text {kink }}$ with the mean-square deviation of the random pinning energy within the area of the defect $U_{r} \sqrt{n_{r} \pi a^{2} r_{d}^{2}}$ and we find

$$
U_{r} \sim \sqrt{\frac{\pi}{n_{r} a^{2}}}\left[U_{0}-2\left(E_{\mathrm{sq}}-E_{\mathrm{hp}}\right)\right] .
$$

From Eq. (34) we see that the $U_{r}$ required to destroy the order in the vortex positions in the square-pinned phase vanishes at $U_{0}=2\left(E_{\mathrm{sq}}-E_{\mathrm{hp}}\right)$, i.e., in the point of the transition between the square-pinned and half-pinned lattices in the absence of disorder. This means that arbitrary weak disorder destroys the regularity in a square vortex lattice in the vicinity of this point, since the energies of pinned and half-pinned lattices are equal to each other there.

We now start to construct a phase diagram of the system in the $U_{0}-U_{r}$ plane for the same concentration of random and regular pins, i.e., $n_{r} a^{2}=1$. The boundary separating pinned and a mixture of pinned and half-pinned lattices is shown by line 1 in Fig. 4 at $a=1$ and $\kappa=5$. This mixed phase still can be considered as pinned, as a whole, by the regular pinning sites array, since there is a strong correlation between the positions of regular sites and the vortices.

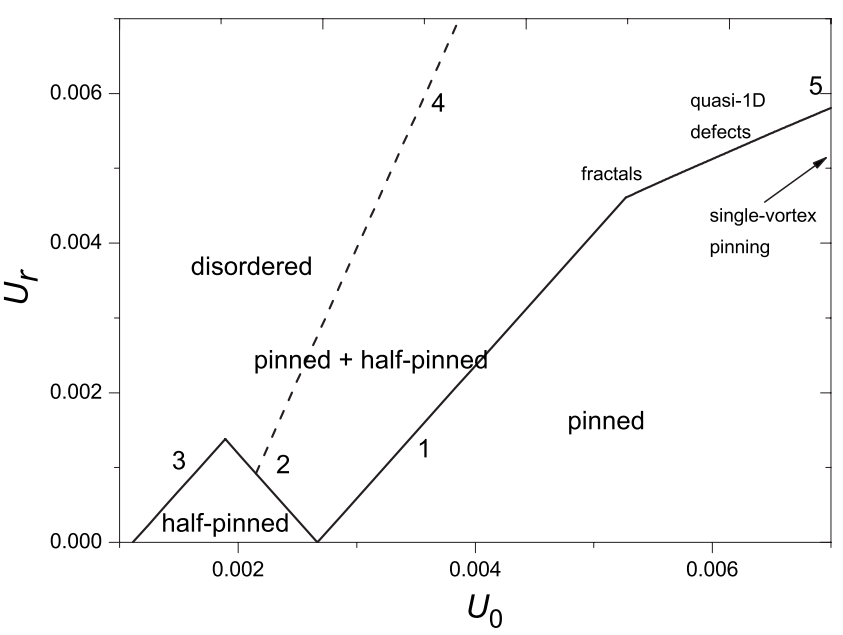

FIG. 4. Schematic phase diagram of the vortex lattice in the plane of the periodic pinning strength $U_{0}$ and the random pinning strength $U_{r}$

Let us now switch to the half-pinned phase. As was explained in Sec. III, the lowest-energy defect in this case corresponds to the row of pinned vortices, which recovers locally square array, as in Fig. 2(b). This row again has to be reconnected. By repeating the derivation which led us to Eq. (34), we obtain the following criterion for $U_{r}$ favoring proliferation of such defects:

$$
U_{r} \sim \sqrt{\frac{\pi}{n_{r} a^{2}}}\left[-U_{0}+2\left(E_{\mathrm{sq}}-E_{\mathrm{hp}}\right)\right] .
$$

It corresponds to the curve 2 in the phase diagram which is basically a mirror image of the boundary given by Eq. (34). It is interesting to note that disorder in this case leads to an unexpected situation, namely, it forces vortices within some regions to be pinned by the regular array of pinning sites. Also note that if we follow along curves 1 and 2, we see that the minimal random pinning strength required for the partial disordering of the vortex lattice is a nonmonotonic function of $U_{0}$. This is again a nontrivial result, since one could expect that the higher $U_{0}$, the higher must be $U_{r}$, which destroys the order in the vortex positions. However, this is not exactly the case, since there is one more factor, i.e., the intervortex interaction, which helps the random pinning sites to destroy order. Thus, at a certain value of $U_{0}$, it is energetically favorable for the system to decrease its energy not by introducing vortex lattice defects but by making a structural phase transition of the vortex lattice, on large scales, into a half-pinned regular phase.

The half-pinned phase can also be destroyed via elastic domains of the deformed triangular lattice; the energy density for the corresponding defect is given by Eq. (19). Thus, the random energy $U_{r}$ generating such domains can be estimated as

$$
U_{r} \sim \sqrt{\frac{\pi}{n_{r} a^{2}}}\left[U_{0}-2\left(E_{\mathrm{hp}}-E_{\mathrm{tr}}\right)\right] .
$$

It is shown as curve 3 in Fig. 4, which separates regions of half-pinned and deformed triangular, i.e., completely disor- 
dered, phase. Let us stress that proliferation of such domains leads to a complete depinning of the vortex lattice from the underlying square array of pinning sites. Curves 2 and 3 intersect in some point and they bound a region in the phase diagram where the half-pinned vortex lattice is stabilized. Of course, this does not mean that there are no defects at all in this region (or in the region of the square pinned lattice), but this implies that their concentration is low. One should also understand that the state, which we call a mixture of pinned and half-pinned phases, contains domains with the deformed triangular lattice as well; but again the concentration of such domains is low. There can be other types of defects, such as vacancies, when one vortex is redistributed to the position which is very far, in terms of lattice period, from its initial position; however, the probability of such events is low, since we are in the regime of collective pinning. Therefore, such events do not contribute significantly to the process of vortex lattice disordering.

Now we discuss how a mixture of pinned and half-pinned phases is finally completely depinned from the regular pinning array. For that we first estimate $U_{r}$ generating domains of triangular lattice inside the square one,

$$
U_{r} \sim 2 \sqrt{\frac{\pi}{n_{r} a^{2}}}\left[U_{0}-\left(E_{\mathrm{sq}}-E_{\mathrm{tr}}\right)\right]
$$

which is depicted as curve 4 in Fig. 4. In reality, before such domains appear in the square phase, it is already disturbed by rows of the half-pinned lattice, so that curve 4 does not describe properly such a transition. The same is true for the curve 3 which describes the transition from the half pinned to the deformed triangular lattice. In other words, curves 3 and 4 must intersect.

From the obtained phase diagram we see that the disordering of the square-pinned lattice in the limit of weak pinning always occurs in two steps. First, some rows of vortices depin and form reconnected elastic chains; their concentration increases with the increase in random pinning strength $U_{r}$. At higher values of $U_{r}$, elastic domains of deformed triangular lattice proliferate in the system leading to a complete disordering of vortex array. If we start from the half-pinned lattice, two scenarios are possible. If the regular pinning strength $U_{0}$ is relatively low, then the order is destroyed in one step due to the proliferation of domains of the deformed triangular lattice. If $U_{0}$ is relatively high, again a two-step disordering can take place. As a first step, an admixture of the square-pinned lattice appear in the system and as a second step, domains of a deformed triangular lattice depin the vortex lattice from the regular pinning array. Note that in experimental work ${ }^{30}$ on macroscopic charged balls on a square array of traps, the formation of domains in the lattice of these balls was also observed. Although there was no artificial randomness in the experimental setup, the disorder was certainly unavoidable. Since energies of three relevant vortex phases, namely, triangular, pinned, and half-pinned ones, are quite close to each other, domains formation can be observed in such experiments.

In Sec. III, we also have described a quasi-1D type of defects, which destroy the order in vortex positions within the same row [see Fig. 2(c)]. The typical area of such a defect is $\sim l_{1 \mathrm{D}} a$, where $l_{1 \mathrm{D}}$ is given by Eq. (27). The energy of the defect is defined in Eq. (28). Thus, for $U_{r}$ leading to the generation of quasi-1D defects, we get

$U_{r} \sim \frac{2}{\sqrt{a}} \frac{2 \pi}{\kappa^{2}}\left(U_{0}-\frac{4 \pi}{\kappa^{2}} e^{-2 \pi}\right)^{3 / 4}\left[K_{0}(a)+\frac{K_{1}(a)}{a}\right]^{1 / 4}$.

Our estimates show that for realistic values of the parameters, $U_{r}$ could become larger than the values corresponding to the phase boundary depicted by lines 2 and 3 . This conclusion is due to the fact that quasi-1D defects represent an intermediate case between the collective pinning of vortices and a regime of single-vortex pinning at high-pinning strengths. The vortex lattice in this case is still an elastic medium, with the intervortex interaction being important in the total balance of the energies. The phase boundary corresponding to such a transition is plotted in Fig. 4 as line 5. Since quasi-1D defects destroy the order in the vortex positions in the same row, a mixture of pinned and half-pinned phases in this case is also characterized by the absence of such an order in the rows of unpinned vortices; moreover, chains of unpinned vortices are now not necessarily reconnected. In this sense, the difference between the quasi-1D disordered phase and a mixture of pinned and half-pinned states is smeared out.

One can expect that the final regime with sufficiently high $U_{0}$ corresponds to zero-dimensional defects, when vortices are depinned individually that is known as the single-vortex pinning regime. Thus, the dimensionality of typical defects leading to the destruction of the order is decreasing continuously from two to zero when the regular pinning strength $U_{0}$ is increasing. At low $U_{0}$, regularity in the vortex positions is destroyed by the appearance of reconnected elastic chains of collectively depinned vortices that are ordered within each chain. At higher $U_{0}$, these chains start to be open, they should become straighter and, therefore, one can expect neighboring chains to connect due to the lack of free space. If we again increase $U_{0}$, chains become quasi-onedimensional elastic rows of finite length, as we have shown schematically in Fig. 2(c). Finally, we are in a single-pinning regime, when each vortex is pinned individually that can be interpreted as chain shrinking toward a single lattice period, since the chain length given by Eq. (27) decreases with the increase in regular pinning strength.

So far only limiting cases of the first stage of vortex lattice disordering have been considered, namely, when we have (i) reconnected chains, i.e., two-dimensional collective defects, (ii) quasi-one-dimensional stripelike collective defects, and (iii) quasi-zero-dimensional individual defects in the regime of a single-vortex pinning. To have a deeper insight into the problem and to understand better how these defects evolve, we perform molecular-dynamics simulations for the regime intermediate between the weak and strong pinning regimes. In Figs. 5-8 we present typical vortex patterns corresponding to an increasing strength in the random pinning potential $U_{r}$ with a constant concentration of random sites, which is set equal to the concentration of regular pins. The initial state of the system (i.e., without random pinning) 


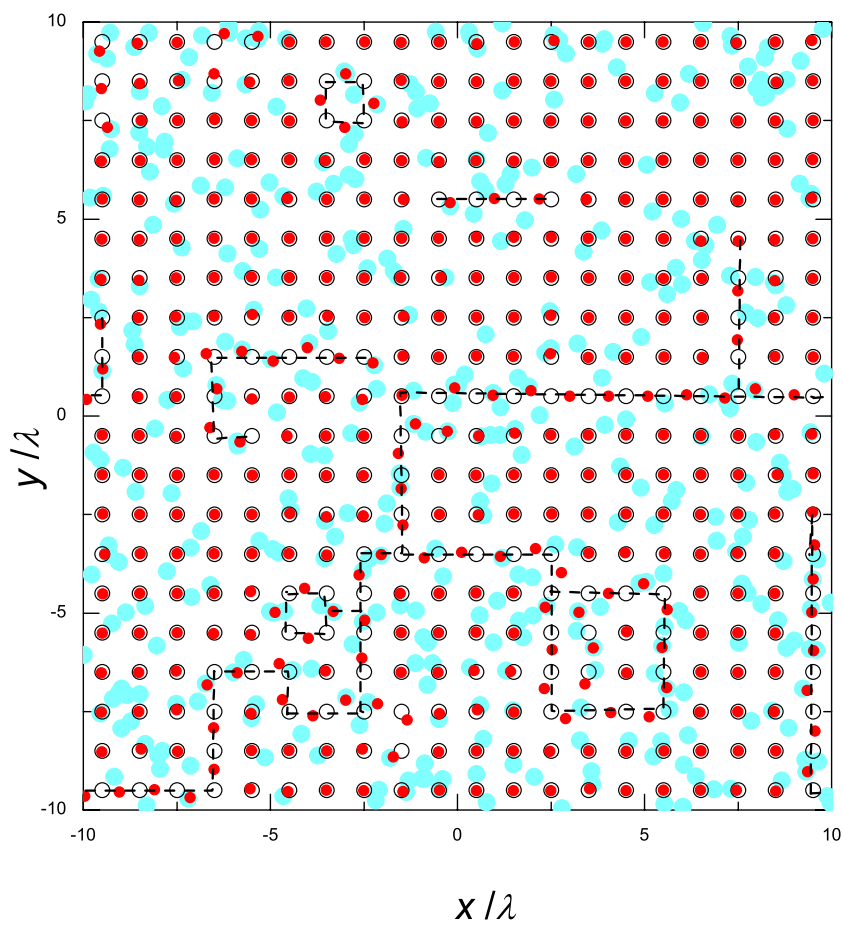

FIG. 5. (Color online) Vortex pattern in the film with regular square and random pinning arrays for $U_{r}=0.72 U_{0}$ obtained in molecular-dynamics simulations. Dashed lines are guides for the eyes indicating positions of chains of unpinned vortices. Irregular blue (light gray) spots represent positions of random pins, red (dark gray) filled circles show positions of vortices, and regular black open circles correspond to periodic pins.

is a square lattice of vortices pinned on regular pins. A simulation region with the sizes $20 \times 20 \lambda$ contains an array of $20 \times 20$ regular pinning arrays and 400 vortices. We use periodic boundary conditions at the boundaries of the simulation region. The value of $U_{0}$ is $0.045 \times 2 \pi / \kappa^{2}$, whereas the intervortex distance is 1 in units of $\lambda$. This value of $U_{0}$ is several times larger than that required for the transition from the square phase to the half-pinned state, i.e., we are indeed in the intermediate regime, where both reconnected and open chains of depinned vortices are expected to appear in the system with increasing $U_{r}$. This is exactly what we see in Fig. 5 at $U_{r}=0.72 U_{0}$, which contains both types of chains, and still there is no domain of a deformed triangular lattice. In general, these elastic chains are packed into finite fractallike structures with a quasi-self-similar topology and dimensionality between one and two. The building blocks of these fractals are represented by parts of rectangles of discrete sizes (i.e., commensurate with the period of the pinning array). If we increase $U_{r}$, as in Fig. 6 for $U_{r}=0.85 U_{0}$, domains of deformed triangular lattice start to appear in the system, and thus they coexist with chains of unpinned vortices. However, these domains somehow suppress the networks of depinned vortices by simply cutting them since there is a competition between these two kinds of defects for a free space in the system. Further increasing $U_{r}$ leads to the increase in the concentration of domains of totally depinned vortices, as indicated in Fig. 7 at $U_{r}=1.28 U_{0}$. It is interesting to note that domains of pinned square vortex lattice turn out to be rather

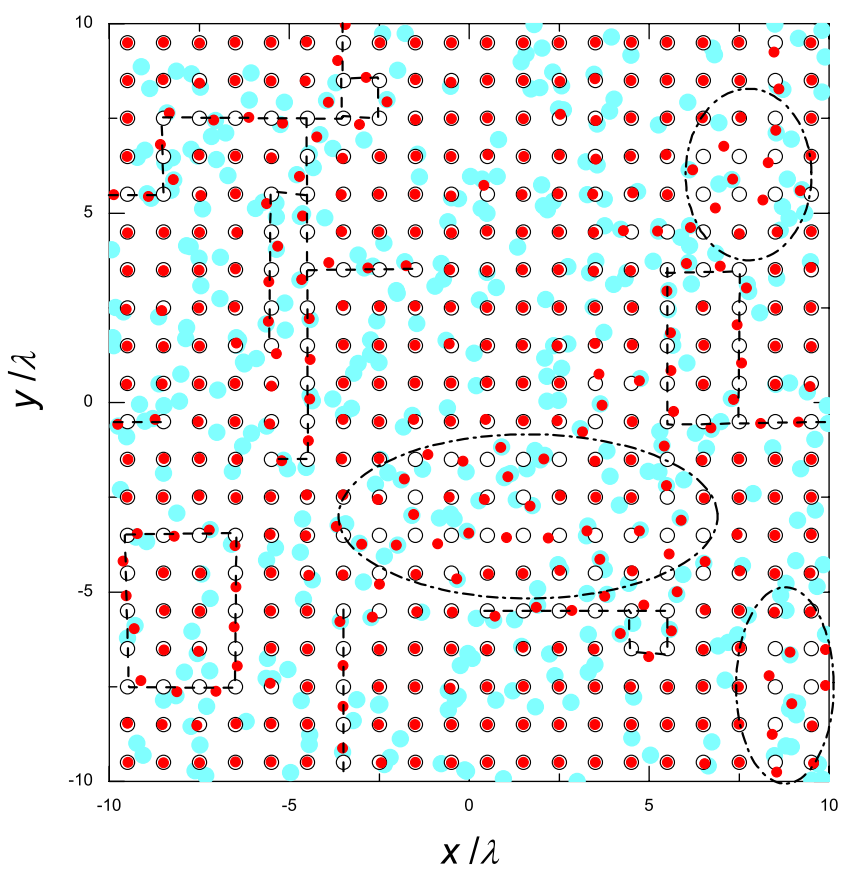

FIG. 6. (Color online) Vortex pattern in the film with regular square and random pinning arrays for $U_{r}=0.85 U_{0}$ obtained in molecular-dynamics simulations. Dashed lines are guides for the eyes indicating positions of chains of unpinned vortices. Dashdotted lines show borders of domains of deformed triangular lattice which is depinned from the underlying regular pinning array. Irregular blue (light gray) spots represent positions of random pins, red (dark gray) filled circles show positions of vortices, and regular black open circles correspond to periodic pins.

robust with respect to the random pinning; some of them survive up to quite large values of $U_{r}$, several times greater than that corresponding to the situation shown in Fig. 7, with the fraction of pinned vortices decreasing slowly with increasing $U_{r}$. Figure 8 shows the final state with quite high value of $U_{r}=5.3 U_{0}$, when almost all the vortices are depinned from the regular pinning array and there is no correlation between the positions of the regular pins and vortices; but there is a pronounced correlation between the positions of the vortices and random pins. Note that typical values of $U_{r}$ leading to the generation of defects are in good qualitative agreement with our analytical predictions.

We also performed simulations for even higher $U_{0}$. We found that indeed the initial defects turn out to be quasi-onedimensional stripes with the characteristic length of few lattice periods rather than long-length and branched structures. Other defects found correspond to higher values of $U_{r}$ and they are more similar to a single-particle imperfections than to regions of elastically distorted media, which are constructed of many interacting particles. In fact, these defects correspond to domains of totally depinned vortices described above, in the limiting case of a single-vortex pinning. Finally, single-vortex defects and stripelike defects also match when the length of the stripes tends to a one lattice period. A general qualitative picture of the evolution of defects is presented in Fig. 9. Thus, the molecular-dynamics simulations enable us to relate defects of various dimensionalities and to better understand their evolution. 


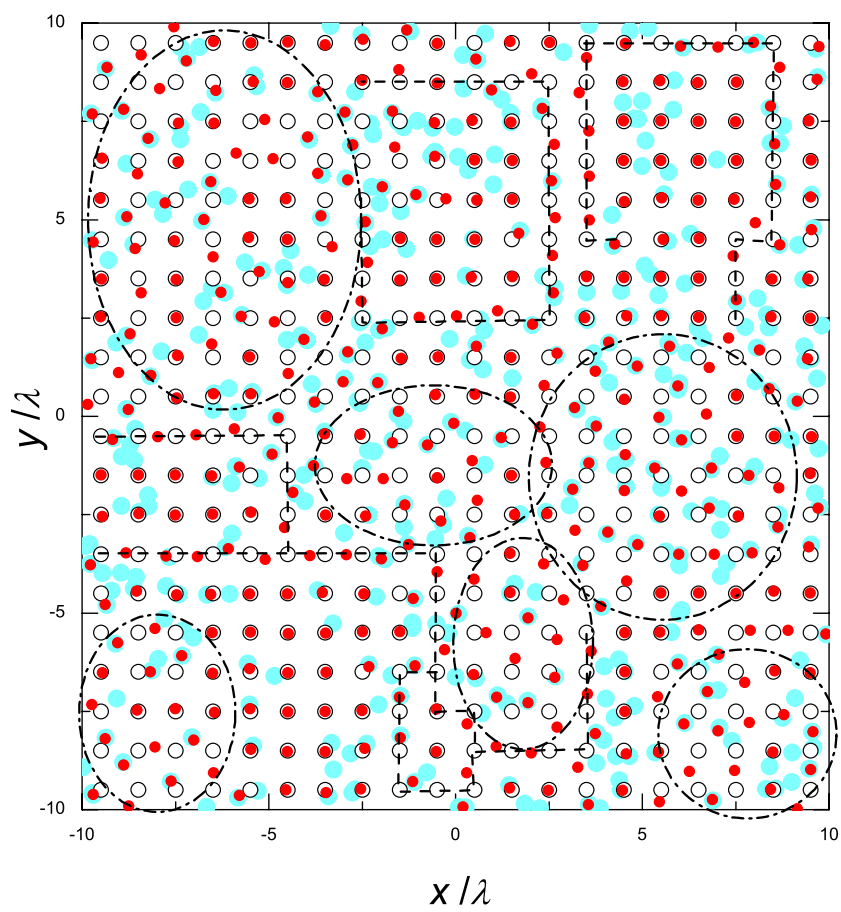

FIG. 7. (Color online) Vortex pattern in the film with regular square and random pinning arrays for $U_{r}=1.28 U_{0}$ obtained in molecular-dynamics simulations. Dashed lines are guides for the eyes indicating positions of chains of unpinned vortices. Dashdotted lines show borders of domains of deformed triangular lattice which is depinned from the underlying regular pinning array. Irregular blue (light gray) spots represent positions of random pins, red (dark gray) filled circles show positions of vortices, and regular black open circles correspond to periodic pins.

\section{CONCLUSIONS}

In this paper, we studied theoretically the effect of disorder on vortex lattices in the presence of a regular square array of pinning sites. We considered a two-dimensional system with the same concentration of regular pinning sites and vortices, i.e., with filling factor 1 . We were mostly focused on a weak pinning regime, when vortex lattice demonstrates collective properties due to the mutual repealing of vortices. The resulting structure of the vortex lattice is determined by the competition between the square symmetry of the regular pinning array, by the triangular symmetry imposed by the vortex-vortex interaction, and by the action of randomness trying to destroy the order in vortex positions. Typical defects of vortex lattices have been identified for some limiting and therefore simple cases and their energies and sizes have been estimated analytically. Molecular-dynamics simulations were also used for exploring regions on phase diagram, which are difficult to approach in an analytical way. Topological defects found are of various dimensionalities including fractional ones having fractal-like structure. We were able to predict analytically two limiting cases of these fractal-like structures, a reconnected chain of unpinned vortices and a straight stripelike defects, while moleculardynamics simulations allowed us to reveal the connection between these limits.

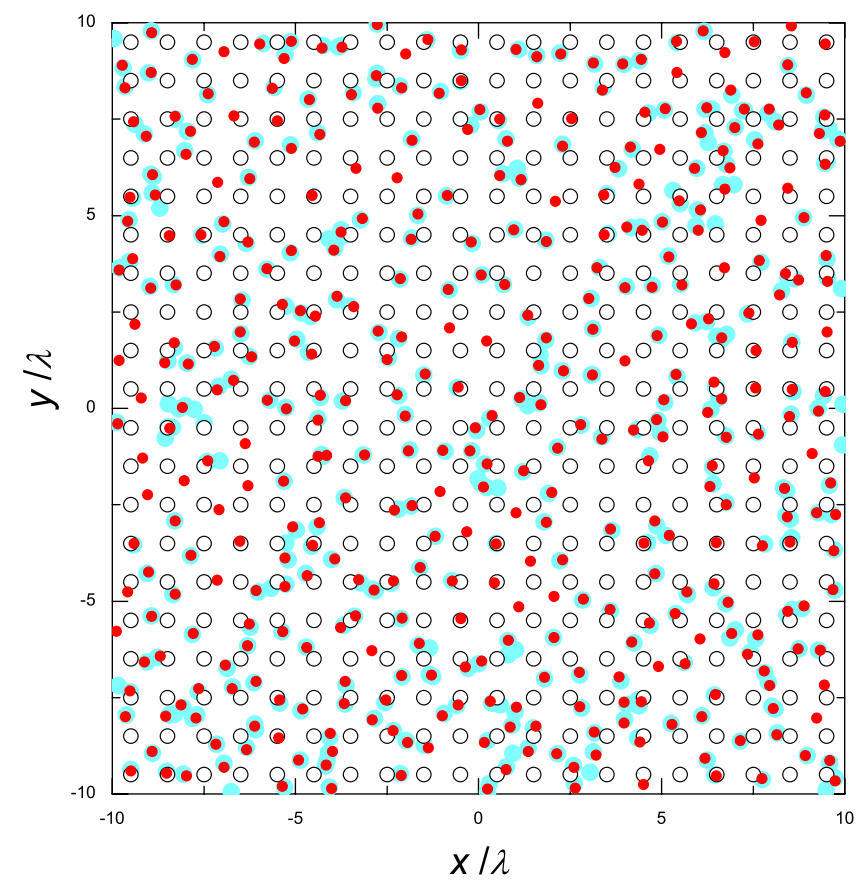

FIG. 8. (Color online) Vortex pattern in the film with regular square and random pinning arrays for $U_{r}=5.3 U_{0}$ obtained in molecular-dynamics simulations. Irregular blue (light gray) spots represent positions of random pins, red (dark gray) filled circles show positions of vortices, and regular black open circles correspond to periodic pins.

We then constructed an approximate phase diagram of the system in the plane of random and regular pinning strengths. We found that disordering of the regularity in vortex positions can occur either in one or two steps depending on the strength of the regular pinning potential. In the former case, domains of deformed triangular lattice start to appear spon-

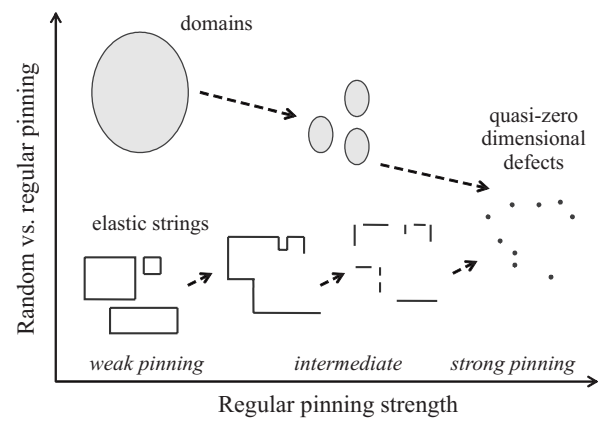

FIG. 9. General picture of the evolution of disorder-induced defects for square-pinned vortex lattice. Lower branch corresponds to the elastic strings of collectively depinned vortices. First, they appear as reconnected chains then chains break and straighten. As a result, they start to overlap and form fractal-like clusters. Finally, they decay to quasi-one-dimensional stripes, which shrink gradually to quasi-zero-dimensional individual defects corresponding to the single-pinning regime. Upper branch shows the evolution of higherenergy elastic defects, which form domains of vortices collectively depinned from regular pins. These domains break into uncorrelated blocks of smaller sizes and finally also shrink to quasi-zerodimensional defects thus matching with the lower branch of defects. 
taneously in the film. In the latter case, in the beginning, reconnected chains of unpinned vortices are generated inside a square lattice (or the opposite: chains of pinned vortices appear inside the half-pinned phase) and only after that again domains of deformed triangular lattice depin the vortex lattice as a whole from the regular pinning array. Both chains and domains are elastic defects with interacting vortices depinned collectively.

We also analyze an intermediate regime for values of regular pinning strength, which are between the regimes of collective and single-vortex pinnings. In this case, the first step of disordering occurs by proliferation of opened, not reconnected, elastic chains of depinned vortices, which can form long fractal-like defects with complicated quasi-selfsimilar topology. Fractals appear due to the process of opening of reconnected chains and their straightening, when different chains start to connect to each other. At the same time, the average length of these defects is going to decrease with the increase in regular pinning strength due to a periodic potential sharpening, so that there is a competition between two tendencies. As a result, chains become straighter and relatively short thus forming quasi-one-dimensional stripes. Finally, for high values of periodic pinning strength, they shrink continuously toward quasi-zero-dimensional defects corresponding to a single-pinning regime. The latter regime is characterized by one-step disordering, i.e., the width of the region on the phase diagram with coexistent chains of unpinned vortices and domains of totally depinned vortices has to vanish with the growth of regular pinning strength, since these two types of defects also match. Thus, we can understand on the same footing all the regimes of disordering starting from a collective pinning to a single-vortex pinning. Our results are applicable not only to vortices in superconductors but also for a broader class of physical systems containing a large number of repealing objects and underlying system of traps: for instance, vortices in ultracold gases in optical lattices, colloids, macroscopic elastic balls interacting via Coulomb forces, and charge and spin-density waves.

\section{ACKNOWLEDGMENTS}

This work was supported by the Flemish Science Foundation (FWO-Vl), the Interuniversity Attraction Poles (IAP) Programme-Belgian State-Belgian Science Policy, and the "Odysseus" program of the Flemish Government and the FWO-Vl. W.V.P. also acknowledges support from the Russian Foundation for Basic Research (Project No. 06-0216691), the President of Russia Programme for Young Scientists, and the Russian Science Support Foundation. V.R.M. acknowledges support from the EU Marie Curie Programme under Contract No. MIF1-CT-2006-040816.

\section{APPENDIX}

Let us assume that for a square-pinned lattice, an infinite kink can have a smooth structure and is directed along the $y$ axis $[x$ and $y$ axes are directed like in Fig. 1(c)], thus separating two regions of vortex lattices shifted by $a$ in $y$ direction with respect to each other, as for usual sine-Gordon systems. ${ }^{40}$ We also denote the kink's length along $x$ as $l_{2 \mathrm{D}}$ $\left(l_{2 \mathrm{D}} \gg a\right)$. There are $l_{2 \mathrm{D}} / a \gg 1$ vortex rows within the kink's length and we denote deviations of vortices in the $n$ 's row along the $y$ direction as $u_{n}$. We also restrict ourselves to lattices with intervortex distances on the order of the penetration depth $\lambda$ ( $a \sim 1$ in dimensionless units). In this case, the dominant contribution to the interaction energy of a given vortex with all other rows of vortices is provided by harmonics with $m=0, \pm 1$, as can be seen from the exponent in the RHS of Eq. (3). This energy is then given by

$$
\begin{aligned}
E_{n} \simeq & \frac{(2 \pi)^{2}}{\kappa^{2} a} \frac{e^{-a}}{1-e^{-a}}+\frac{2 \pi}{\kappa^{2}} e^{-2 \pi}\left[\cos \left(\frac{2 \pi\left(u_{n+1}-u_{n}\right)}{a}\right)\right. \\
& \left.+\cos \left(\frac{2 \pi\left(u_{n}-u_{n-1}\right)}{a}\right)\right] .
\end{aligned}
$$

Now we can expand the RHS of Eq. (A1) in terms of $\Delta u_{n} / a$. To find the energy of the kink, we have to sum the contributions from all the rows within the kink. But instead it is convenient to switch from summation to integration with the simultaneous exchange of $\Delta u_{n} / a$ by $\frac{d u}{d x}$, i.e., to use a continuous limit and introduce smoothly varying deformation field of the vortex lattice. This method is applicable for sineGordon systems, ${ }^{40}$ and we repeat the same arguments for the system at hand. Finally, for the $u$-dependent part of the vortex-vortex energy of the kink (per length of the kink in $y$ direction), we have

$$
E_{\mathrm{kink}}^{(v v)}=-\frac{(2 \pi)^{3}}{\kappa^{2} a^{2}} e^{-2 \pi} \int\left(\frac{d u}{d x}\right)^{2} d x .
$$

The derivative $\frac{d u}{d x}$ can be estimated as $\frac{d u}{d x} \approx \frac{a}{l_{2 D}}$ and this leads us to the following expression:

$$
E_{\mathrm{kink}}^{(v v)} \approx-\frac{(2 \pi)^{3}}{\kappa^{2}} e^{-2 \pi} \frac{1}{l_{2 \mathrm{D}}} .
$$

There is also a contribution to the kink energy coming from the regular pinning potential. It appears due to the fact that vortex rows within this kink are depinned. In the limit of small potential wells $\sigma \ll a$, this energy (per length of the kink in $y$ direction) is given by

$$
E_{\mathrm{kink}}^{(v p)} \approx U_{0} \frac{l_{2 \mathrm{D}}}{a^{2}} .
$$

For the total energy of the kink, we thus have

$$
E_{\mathrm{kink}}=E_{\mathrm{kink}}^{(v v)}+E_{\mathrm{kink}}^{(v p)} \approx-\frac{(2 \pi)^{3}}{\kappa^{2}} e^{-2 \pi} \frac{1}{l_{2 \mathrm{D}}}+U_{0} \frac{l_{2 \mathrm{D}}}{a^{2}} .
$$

If we now try to minimize the kink energy given by Eq. (A5) with respect to the kink's length, we immediately see that $l_{2 \mathrm{D}}=0$ is the lowest-energy solution. Of course, in reality $l_{2 \mathrm{D}}$ cannot be shorter than $a$ and $l_{2 \mathrm{D}}=a$ is a true solution. This conclusion is based on the physical fact that a square lattice of free interacting vortices is unstable in the absence of pinning, leading to a negativeness of "elastic constant," in contrast to the sine-Gordon and related systems. ${ }^{40}$ 
${ }^{1}$ M. Baert, V. V. Metlushko, R. Jonckheere, V. V. Moshchalkov, and Y. Bruynseraede, Phys. Rev. Lett. 74, 3269 (1995).

${ }^{2}$ V. V. Moshchalkov, M. Baert, V. V. Metlushko, E. Rosseel, M. J. Van Bael, K. Temst, R. Jonckheere, and Y. Bruynseraede, Phys. Rev. B 54, 7385 (1996).

${ }^{3}$ A. T. Fiory, A. F. Hebard, and S. Somekh, Appl. Phys. Lett. 32, 73 (1978).

${ }^{4}$ A. M. Castellanos, R. Wördenweber, G. Ockenfuss, A. v. d. Hart, and K. Keck, Appl. Phys. Lett. 71, 962 (1997); R. Wördenweber, P. Dymashevski, and V. R. Misko, Phys. Rev. B 69, 184504 (2004).

${ }^{5}$ J. Eisenmenger, Z.-P. Li, W. A. A. Macedo, and I. K. Schuller, Phys. Rev. Lett. 94, 057203 (2005).

${ }^{6}$ J. E. Villegas, E. M. Gonzalez, M. I. Montero, I. K. Schuller, and J. L. Vicent, Phys. Rev. B 68, 224504 (2003); M. I. Montero, J. J. Akerman, A. Varilci, and I. K. Schuller, Europhys. Lett. 63, 118 (2003).

${ }^{7}$ J. E. Villegas, S. Savel'ev, F. Nori, E. M. Gonzalez, J. V. Anguita, R. García, and J. L. Vicent, Science 302, 1188 (2003).

${ }^{8}$ L. Van Look, B. Y. Zhu, R. Jonckheere, B. R. Zhao, Z. X. Zhao, and V. V. Moshchalkov, Phys. Rev. B 66, 214511 (2002).

${ }^{9}$ A. V. Silhanek, S. Raedts, M. Lange, and V. V. Moshchalkov, Phys. Rev. B 67, 064502 (2003).

${ }^{10}$ M. Kemmler, C. Gürlich, A. Sterck, H. Pöhler, M. Neuhaus, M. Siegel, R. Kleiner, and D. Koelle, Phys. Rev. Lett. 97, 147003 (2006).

${ }^{11}$ A. V. Silhanek, W. Gillijns, V. V. Moshchalkov, B. Y. Zhu, J. Moonens, and L. H. A. Leunissen, Appl. Phys. Lett. 89, 152507 (2006).

${ }^{12}$ F. Nori, Science 271, 1373 (1996); C. Reichhardt, C. J. Olson, J. Groth, S. Field, and F. Nori, Phys. Rev. B 52, 10441 (1995); 53, R8898 (1996); C. Reichhardt, J. Groth, C. J. Olson, S. B. Field, and F. Nori, ibid. 54, 16108 (1996).

${ }^{13}$ C. Reichhardt, C. J. Olson, and F. Nori, Phys. Rev. B 57, 7937 (1998).

${ }^{14}$ C. Reichhardt, C. J. Olson, and F. Nori, Phys. Rev. Lett. 78, 2648 (1997); Phys. Rev. B 58, 6534 (1998).

${ }^{15}$ V. R. Misko, S. Savel'ev, A. L. Rakhmanov, and F. Nori, Phys. Rev. Lett. 96, 127004 (2006); Phys. Rev. B 75, 024509 (2007).

${ }^{16}$ G. R. Berdiyorov, M. V. Milošević, and F. M. Peeters, Phys. Rev. B 74, 174512 (2006).

${ }^{17}$ G. R. Berdiyorov, M. V. Milošević, and F. M. Peeters, Phys. Rev. B 76, 134508 (2007).

${ }^{18}$ V. Misko, S. Savel'ev, and F. Nori, Phys. Rev. Lett. 95, 177007 (2005); V. R. Misko, S. Savel'ev, and F. Nori, Phys. Rev. B 74, 024522 (2006); Physica C 437-438, 213 (2006).

${ }^{19}$ V. N. Rudko, O. N. Shevtsova, and S. V. Shiyanovsky, Fiz. Nizk.
Temp. 22, 1314 (1996).

${ }^{20}$ C. Reichhardt, C. J. Olson, R. T. Scalettar, and G. T. Zimanyi, Phys. Rev. B 64, 144509 (2001).

${ }^{21}$ W. V. Pogosov, A. L. Rakhmanov, and V. V. Moshchalkov, Phys. Rev. B 67, 014532 (2003).

${ }^{22}$ Q. H. Chen, G. Teniers, B. B. Jin, and V. V. Moshchalkov, Phys. Rev. B 73, 014506 (2006).

${ }^{23}$ C. Reichhardt and C. J. Olson Reichhardt, Phys. Rev. B 76, 064523 (2007).

${ }^{24}$ V. Zhuravlev and T. Maniv, Phys. Rev. B 68, 174507 (2003).

${ }^{25}$ G. R. Berdiyorov, M. V. Milošević, and F. M. Peeters, Physica C 468, 809 (2008).

${ }^{26}$ J. W. Reijnders and R. A. Duine, Phys. Rev. Lett. 93, 060401 (2004).

${ }^{27}$ J. W. Reijnders and R. A. Duine, Phys. Rev. A 71, 063607 (2005).

${ }^{28}$ H. Pu, L. O. Baksmaty, S. Yi, and N. P. Bigelow, Phys. Rev. Lett. 94, 190401 (2005).

${ }^{29}$ S. Tung, V. Schweikhard, and E. A. Cornell, Phys. Rev. Lett. 97, 240402 (2006).

${ }^{30}$ G. Coupier, M. Saint Jean, and C. Guthmann, Phys. Rev. B 75, 224103 (2007).

${ }^{31}$ K. Mangold, P. Leiderer, and C. Bechinger, Phys. Rev. Lett. 90, 158302 (2003).

${ }^{32}$ D. Deb and H. H. von Grunberg, J. Phys.: Condens. Matter 20, 245104 (2008).

${ }^{33}$ A. A. Zhukov, H. Kupfer, G. K. Perkins, A. D. Caplin, T. Wolf, K. I. Kugel, A. L. Rakhmanov, M. G. Mikheev, V. I. Voronkova, M. Klaser, and H. Wuhl, Phys. Rev. B 59, 11213 (1999).

${ }^{34}$ K. I. Kugel, A. L. Rakhmanov, and A. A. Zhukov, Physica C 334, 203 (2000).

${ }^{35}$ I. F. Voloshin, A. V. Kalinov, L. M. Fisher, K. I. Kugel, and A. L. Rakhmanov, JETP 84, 1177 (1997).

${ }^{36}$ K. Harada, O. Kamimura, H. Kasai, T. Matsuda, A. Tonomura, and V. V. Moshchalkov, Science 274, 1167 (1996).

${ }^{37}$ Y. Togawa, K. Harada, T. Akashi, H. Kasai, T. Matsuda, F. Nori, A. Maeda, and A. Tonomura, Phys. Rev. Lett. 95, 087002 (2005).

${ }^{38}$ I. V. Grigorieva, W. Escoffier, V. R. Misko, B. J. Baelus, F. M. Peeters, L. Y. Vinnikov, and S. V. Dubonos, Phys. Rev. Lett. 99, 147003 (2007).

${ }^{39}$ H. J. Zhao, V. R. Misko, F. M. Peeters, S. Dubonos, V. Oboznov, and I. V. Grigorieva, Europhys. Lett. 83, 17008 (2008).

${ }^{40}$ P. M. Chaikin and T. C. Lubensky, Principles of Condensed Matter Physics (Cambridge University Press, Cambridge, 1995).

${ }^{41}$ G. Blatter, M. V. Feigel'man, V. B. Geshkenbein, A. I. Larkin, and V. M. Vinokur, Rev. Mod. Phys. 66, 1125 (1994). 